\title{
All-Sky-ASTROGAM: a MeV Companion for Multimessenger Astrophysics
}

\author{
A. De Angelis ${ }^{1,2}$, V. Tatischeff ${ }^{3}$, M. Mallamaci ${ }^{1, *}$, R. Rando ${ }^{1,+}$, , M. Tavani ${ }^{4}$, \\ U. Oberlack ${ }^{5}$, R. Walter ${ }^{6}$, G. Ambrosi ${ }^{7}$, A. Argan ${ }^{8}$, P. von Balmoos ${ }^{9}$, D. Bastieri ${ }^{1,10}$, \\ E. Bernardini ${ }^{1,11}$, S. Brandt ${ }^{12}$, A. Bulgarelli ${ }^{13}$, A. Bykov ${ }^{14}$, V. Fioretti $^{13}$, I. A. Grenier ${ }^{15}$, \\ L. Hanlon ${ }^{16}$, D. Hartmann ${ }^{17}$, M. Hernanz ${ }^{18}$, G. Kanbach ${ }^{19}$, I. Kuvvetli ${ }^{12}$, P. Laurent ${ }^{20}$, \\ M. Mariotti ${ }^{1}$, M. N. Mazziotta ${ }^{21}$, J. Mc Enery ${ }^{22}$, S. Mereghetti ${ }^{23}$, A. Morselli ${ }^{24}$, \\ K. Nakazawa ${ }^{25}$, M. Pearce ${ }^{26}$, E. Prandini ${ }^{1}$, J. Rico ${ }^{27}$, R. Curado da Silva ${ }^{28}$, X. Wu ${ }^{6}$, \\ A. Zdziarski ${ }^{29}$, A. Zoglauer $^{30}$ on behalf of the All-Sky-ASTROGAM Collaboration
}

${ }^{1}$ Padova University and INFN, Italy, ${ }^{2}$ INAF Padova, Italy and Udine University, Italy and LIP, Lisboa, Portugal, ${ }^{3}$ CSNSM, CNRS and University of Paris Sud, Orsay, France, ${ }^{4}$ INAF/IAPS, Roma, Italy, ${ }^{5}$ Institute of Physics and PRISMA Excellence Cluster, University Mainz, Germany, ${ }^{6}$ University of Geneva, Switzerland, ${ }^{7}$ INFN Perugia, Italy, ${ }^{8}$ INAF Headquarters, Roma, Italy, ${ }^{9}$ IRAP Toulouse, France, ${ }^{10}$ Guangzhou University, China, ${ }^{11}$ DESY Zeuthen, Germany, ${ }^{12}$ DTU Space, National Space Institute, Technical University of Denmark, ${ }^{13}$ INAF/OAS-Bologna, Italy, ${ }^{14}$ Ioffe Institute, St.Petersburg, Russia, ${ }^{15}$ Lab. AIM,CEA-IRFU/CNRS/Univ. Paris Diderot, C.E.A. Saclay, France, ${ }^{16}$ University College Dublin, Ireland, ${ }^{17}$ Clemson University, USA, ${ }^{18}$ CSIC-IEEC, Campus UAB, Barcelona, Spain, ${ }^{19}$ MPI, Garching, Germany, ${ }^{20}$ Lab. APC, CEA/DRF CNRS, Univ. Paris Diderot, Paris, France, ${ }^{21}$ INFN Bari, Italy, ${ }^{22}$ NASA Goddard Space Flight Center, MD, USA, ${ }^{23}$ INAF/IASF, Milano, Italy, ${ }^{24}$ INFN Roma Tor Vergata, Italy, ${ }^{25}$ University of Tokyo, Japan, ${ }^{26} \mathrm{KTH}$, Stockholm, Sweden, ${ }^{27}$ IFAE-BIST, Barcelona, Spain, ${ }^{28}$ LIP, Portugal, ${ }^{29}$ Polish Academy of Sciences, Warszawa, Poland, ${ }^{30}$ Berkeley, Space Sciences Laboratory, USA

*manuela.mallamaci@pd.infn.it

${ }^{+}$presenter

In the era of multi-messenger astronomy it is of paramount importance to have in space a gammaray monitor capable of detecting energetic transients in the energy range from $0.1 \mathrm{MeV}$ to a few hundred MeV, with good imaging capabilities. The All-Sky-ASTROGAM mission proposal aims to place into an L2 orbit a gamma-ray instrument $(\sim 100 \mathrm{~kg})$ dedicated to fast detection, localization, and gamma-ray spectroscopy of flaring and merging activity of compact objects in the Universe, with unprecedented sensitivity and polarimetric capability in the $\mathrm{MeV}$ range. The instrument is based on the ASTROGAM concept, which combines three detection systems of space-proven technology: a silicon tracker in which the cosmic gamma rays undergo Compton scattering or a pair conversion, a scintillation calorimeter to absorb and measure the energy of the secondary particles, and an anticoincidence system to veto the prompt reaction background induced by charged particles. The gamma-ray imager and the platform will be connected through a boom and will have almost no occultation, making possible a continuous monitoring of every single gamma-ray source in the sky during the entire mission lifetime.

36th International Cosmic Ray Conference -ICRC2019-

July 24th - August 1st, 2019

Madison, WI, U.S.A. 


\section{Introduction}

Multimessenger astronomy has started during the last years thanks to the detection of gravitational waves (GWs) from binary mergers and of a neutrino from the Active Galactic Nucleus (AGN) TXS $0506+056$. Both events highlight the importance of the MeV range as the perfect companion for multimessenger astronomy. The binary neutron-star (NS-NS) merger generating the event GW170817 and the corresponding gamma-ray signal detected by the Fermi GBM and INTEGRAL [1] have shown that the electromagnetic cutoff of this class of mergers is likely in the (tens of) $\mathrm{MeV}$ range. In addition, this energy band is ideally suited for the inquiry of the acceleration mechanisms in AGN, first of all because emission at higher gamma-ray energies may be absorbed. Secondly, the specific contribution from photo-pair-production by high-energy cosmic nuclei is a critical discriminant in the soft gamma-ray band, as demonstrated in [2].

It is therefore clear that the presence of an instrument with imaging capabilities that monitors the high-energy Universe in the MeV domain is fundamental. All-Sky-ASTROGAM has been proposed with this aim as an instrument dedicated to fast detection of transient phenomena, localization, and gamma-ray spectroscopy of flaring and merging activity of compact objects in the Universe, with excellent polarimetric capabilities. The instrument is a nearly $4 \pi$ gamma-ray imager attached to a deployable boom. It is based on the ASTROGAM concept [3, 4]: a telescope made of thin silicon tracking planes and a scintillation calorimeter to image the Compton interaction and the pair production by gamma rays. In this paper, the science case of All-Sky-ASTROGAM is summarised (Sec. 2), the instrument and its performance are described (Sec. 3). A particular attention is given to the possible joint detection of gamma rays/GWs (Sec. 4.1) and gamma rays/neutrinos (Sec. 4.2).

\section{The science case}

All-Sky-ASTROGAM is conceived to provide unique and complementary gamma-ray data of signicant interest to a broad astronomical community, in a decade of powerful observatories for multiwavelength astronomy and for the detection of gravitational waves, neutrinos and ultra-highenergy cosmic rays (UHECRs). The mission would open the $\mathrm{MeV}$ region for exploration, with an improvement close to one order of magnitude in sensitivity (Fig. 1) compared to the current state of the art, much of which was derived from the COMPTEL instrument more than two decades ago. All-Sky-ASTROGAM would also achieve an improvement in terms of source localization and energy resolution, and would allow to measure the contribution to the radiation of the Universe in an unknown range [5]. At higher energies, reaching almost one $\mathrm{GeV}$, the sensitivity of AllSky-ASTROGAM can reveal the transition from nuclear processes to those involving electro- and hydro-dynamical, magnetic and gravitational interactions. It will complement other observatories, being:

- a unique gamma-ray monitor for astrophysical transients with large FoV (almost $4 \pi$ sr) and optimal sensitivity to detect bright and intermediate flux sources, like Gamma-Ray Bursts(GRBs), AGN, Galactic sources, in the range $0.1 \mathrm{MeV}-500 \mathrm{MeV}$ at different timescales (seconds, hours, days, weeks); 


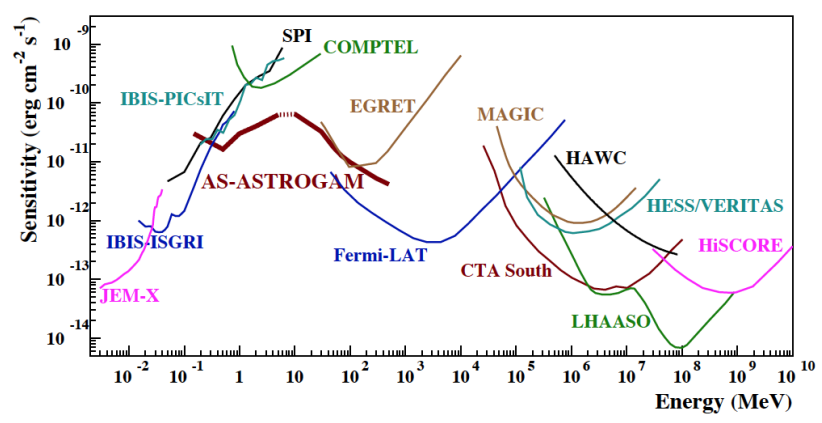

Figure 1: Point source continuum sensitivity of different $\mathrm{X}$ - and gamma-ray instruments. The curves for INTEGRAL/JEM-X, IBIS (ISGRI and PICsIT), and SPI are for an observing time $T_{\mathrm{obs}}=1 \mathrm{Ms}$. The COMPTEL and EGRET sensitivities are given for the observing time accumulated during the whole duration of the CGRO mission ( $T_{\mathrm{obs}} \sim 9$ years). The Fermi-LAT sensitivity is for a high Galactic latitude source and for a mission duration of 10 years. For MAGIC, VERITAS, and CTA, the sensitivities are given for $T_{\mathrm{obs}}$ $=50$ hours. For HAWC $T_{\mathrm{obs}}=5 \mathrm{yr}$, for LHAASO $T_{\mathrm{obs}}=1 \mathrm{yr}$, and for HiSCORE $T_{\mathrm{obs}}=1000 \mathrm{~h}$. The All-Sky-ASTROGAM sensitivity is for an effective exposure of 1 year of a source at high Galactic latitude.

- a unique instrument for the New Astronomy of gravitational waves and neutrinos being able to detect prompt hard X-ray/gamma-ray emission from coalescing NS-NS and NS-BH binaries (with a capability of fast alerts to the community) and $v$ counterparts;

- a unique instrument to improve our knowledge of our Galaxy in an energy range crucial for studying nucleosynthesis and chemical evolution of the Milky Way.

\section{The instrument}

All-Sky-ASTROGAM is shown in Figure 2. It will be attached to a 6 meters-long boom, which will be deployed when the spacecraft reaches its operational orbit (lagrangian point L2), to reduce the gamma-ray shadow cast by the satellite platform on the telescope and decrease the instrument background induced by cosmic-ray interactions with the platform materials.

Interactions of photons with matter in the All-Sky-ASTROGAM energy range is dominated by Compton scattering from $100 \mathrm{keV}$ up to about $15 \mathrm{MeV}$ in silicon, and by electron-positron pair production in the field of a target nucleus at higher energies. All-Sky-ASTROGAM maximises its efficiency for imaging and spectroscopy of energetic gamma-rays by using both processes. The scientific payload consists in particular of three main detectors:

- A silicon Tracker in which the cosmic gamma rays undergo a first Compton scattering or a pair conversion; it is based on the technology of double sided Si strip detectors to measure the energy and the 3D position of each interaction with an excellent energy and spatial resolution;

- A 3D-imaging Calorimeter to absorb and measure the energy of the secondary particles; it is made of an array of small scintillation crystals (784 CsI (Tl) bars of $10 \times 10 \times 50 \mathrm{~mm}^{3}$ ) read out by silicon drift photodetectors to achieve the required energy resolution $(4.5 \%$ at $662 \mathrm{keV})$;

- An Anticoincidence (AC) system covering the six faces of the instrument to veto the promptreaction background (as opposed to the delayed background due to the instrument material 
activation) induced by charged cosmic-ray particles; it is designed with plastic scintillator tiles covering the instrument to detect charged relativistic particles with an efficiency exceeding $99.99 \%$.

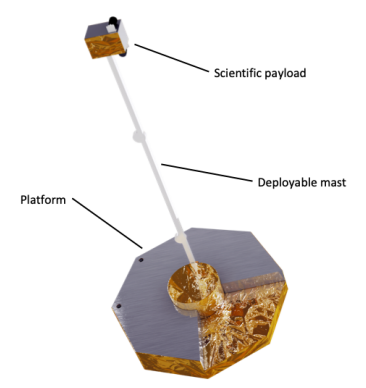

Figure 2: The All-Sky-ASTROGAM satellite.

The performances of All-Sky-ASTROGAM are shown in Fig. 3. The sensitivity of All-SkyASTROGAM is shown in Fig. 1. All-Sky-ASTROGAM will be able also to perform unprecedented polarization measurements thanks to the fine 3D position resolution of both the Si Tracker and the Calorimeter, as well as the light mechanical structure of the Tracker, which is devoid of any heavy absorber in the detection volume.

\section{Multimessenger astronomy with All-Sky-ASTROGAM}

\subsection{Electromagnetic counterparts to gravitational wave transients}

Joint GW and EM observations have a key role for a more complete knowledge of the sources
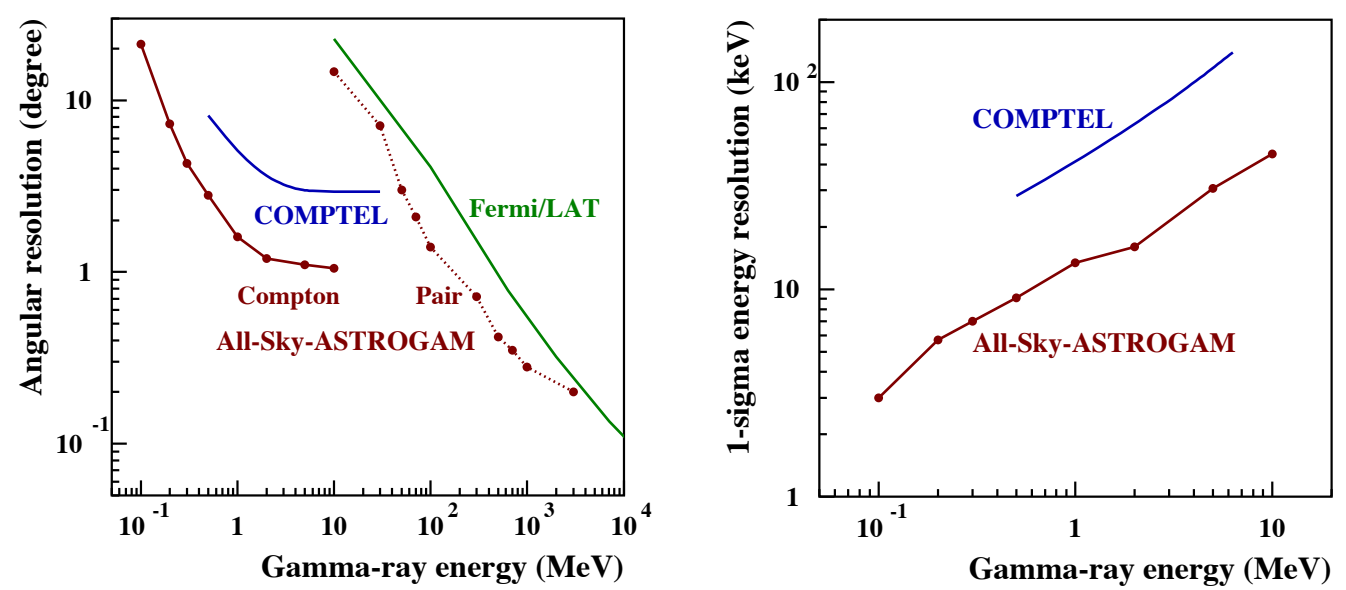

Figure 3: Left panel - All-Sky-ASTROGAM on-axis angular resolution compared to that of COMPTEL and Fermi-LAT. In the Compton domain, the presented performance of All-Sky-ASTROGAM and COMPTEL is the FWHM of the angular resolution measure (ARM). In the pair domain, the point spread function (PSF) is the $68 \%$ containment radius for a $30^{\circ}$ point source. The Fermi-LAT PSF is from the Pass 8 analysis (release 2 version 6) and corresponds to the FRONT and PSF event type. Right panel $-1 \sigma$ energy resolution of COMPTEL and All-Sky-ASTROGAM in the Compton domain after event reconstruction and selection on the ARM. 
and their environments. From one side, GW signals provide information about the physics of the source such as, e.g., the mass and the distance; on the other hand, the identification of the possible EM counterpart pinpoints the location of the burst, possibly identifying the host galaxy and properly defining the astrophysical context.

The long-standing quest for the observation of GWs simultaneously with gamma rays met with success when LIGO and Virgo detected GW170817, the first signal from the coalescence of two NS, $1.7 \mathrm{~s}$ before the gamma-ray signal detected by the Fermi GBM and by INTEGRAL. Thanks to a dedicated follow-up campaign, electromagnetic (EM) counterparts to GW170817 were found in the visible, X-ray and radio bands. Also NS-black hole (BH) mergers, not observed yet, are expected to have an associated EM emission. The energy cutoff for the gamma-ray counterparts on NS-NS and NS-BH mergers is expected to fall in the region of tens of MeV, well appropriate for All-SkyASTROGAM [4]. The detection of the gamma-ray counterpart with All-Sky-ASTROGAM will help to understand if also NS-BH systems are progenitors of short GBRs and to characterise the astrophysical properties of the source. These results will also improve our knowledge of the stellar population of our Galaxy, with a particular focus on the progenitor of merging binary systems.

Another interesting aspect regards the off-axis emission. The EM emission from short GRBs is believed to be beamed. However, the majority of NS-NS merger events will correlate to off-axis short GRBs, as suggested by simple geometrical arguments based on the presumable small opening angle $\theta \sim 10^{\circ}$ of the jet [6]. Taking into consideration that the observed flux from on-axis GRBs is enhanced by beaming, off-axis GRBs flux is dramatically weaker and very sensitive gamma-ray instruments are needed to reveal nearby off-axis GRBs associated to GW events.

From the observational point of view, the follow-up of GW170817 conducted in optical, IR and UV revealed the presence of an EM counterpart with emission consistent with a kilonova, while $\mathrm{X}$-ray and radio data are interpreted as due to an off-axis afterglow emission [7].

Polarization is expected if the jet launching is driven by magnetic energy and depending on the magnetic field configuration. Off-axis observations can introduce an anisotropy that enhances the degree of polarization $[8,9]$. In case a high-energy MeV-GeV component is observed, polarization can help to discriminate between different emission processes such as Inverse-Compton (IC) emission of leptons (no polarization) and synchrotron polarized emission from hadrons. Measurement of the gamma-ray polarization in GW triggered events could provide a new tool for the interpretation of the GW/EM emission. The possible detection of polarization from GRB associated to GW events with All-Sky-ASTROGAM shall have a tremendous impact on the interpretation of the formation of the jet and radiation mechanisms. The simulation of All-Sky-ASTROGAM predicts that a number between 0.2 and 6 NS-NS mergers per year will be detected within 30 degrees (see, e.g., [10]) of the jet axis in coincidence with a GW detection, after the incorporation of KAGRA and INDIGO in the GW network.

All-Sky-ASTROGAM will also play a key role in the multiwavelength study of GW events: in fact, its large FoV will maximize the detection probability and provide accurate sky localization $(<1$ square degree at $1 \mathrm{MeV}$ ), thus allowing the follow-up of the GW events by other telescopes. This capability will be crucial for the identification and the multiwavelength characterization of the GW progenitor and of its host galaxy. Localizations will also guide wide-field follow-up observers in afterglow detection and redshift measurement, and allow long-baseline observations. Figure 4 illustrates the superior sensitivity of All-Sky-ASTROGAM (compared to INTEGRAL) to detect the 


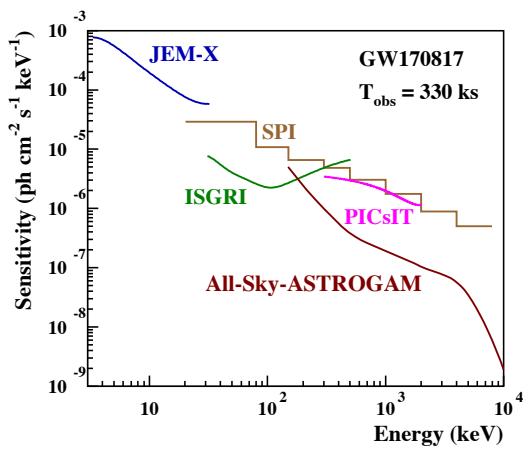

Figure 4: Continuum sensitivity reached in the INTEGRAL targeted follow-up observation of GW170817, compared to the corresponding sensitivities of All-Sky-ASTROGAM. All sensitivities are shown for a total exposure of $330 \mathrm{ks}$. Adapted from Fig. 5 of Ref. [11].

continuum emission expected from the kilonova following a merger event like GW170817. Kilonovae are thought to be primarily powered by the radioactive decay of r-process nuclei synthesized in the merger outflows, and All-Sky-ASTROGAM could detect the predicted gamma-ray emission [12] up to a maximum distance of $\sim 5-6 \mathrm{Mpc}$.

\subsection{Synergies with neutrino telescopes}

Astrophysical high-energy neutrinos at $\mathrm{TeV}-\mathrm{PeV}$ energies are thought to be mainly generated by the decay of charged pions produced in inelastic photo-hadronic $(p \gamma)$ or hadronuclear $(p p)$ processes, involving protons $\sim 20$ times more energetic than the resulting neutrinos. A diffuse flux of cosmic neutrinos has been detected by IceCube [13], the sources of which are still unknown. Identifying those sources and their association with EM counterparts would provide unique insights into the long-standing problem of the origin of cosmic rays. Many astrophysical source classes have been suggested as responsible for the IceCube signal, like star-forming and/or star-burst galaxies, GRBs, or AGN. Galactic sources like microquasars are also expected to be emitters of astrophysical neutrinos. Neutrinos could be emitted during flaring events. Neutrino telescopes like IceCube operate extensive programs of real-time multi-wavelength follow-up, enabling the search for an EM counterpart of astrophysical neutrino candidates by generating real-time alerts. The first compelling evidence of an EM counterpart was recorded on September 2017, when theFermi-LAT and MAGIC observed enhanced gamma-ray emission from a blazar positionally consistent with the neutrino IC170922A [2, 14, 15].

In Fig. 5, the sensitivity of All-Sky-ASTROGAM in 7 days (the time in which MAGIC observed an enhanced signal associated to the blazar TXS 0506+056) is compared to the spectral energy distribution of TXS 0506+056 [2]. Simultaneous time-resolved multi-wavelength information of variable objects at a daily-timescale (as TXS 0506+056) is fundamental for pinpointing the emission mechanism, but is currently not feasible with Fermi. All-Sky-ASTROGAM will have a higher sensitivity than Fermi-LAT over the overlapping energy range. This would have allowed to resolve the state of the source associated to the IceCube neutrino IC170922A. In addition, the energy range not yet dominated by IC electron emission can be covered by All-Sky-ASTROGAM. There, it can be expected to be easier to single out hadronic components and constrain the efficiency as neutrino source. The follow-up abilities of All-Sky-ASTROGAM, given by its large field of view and local- 


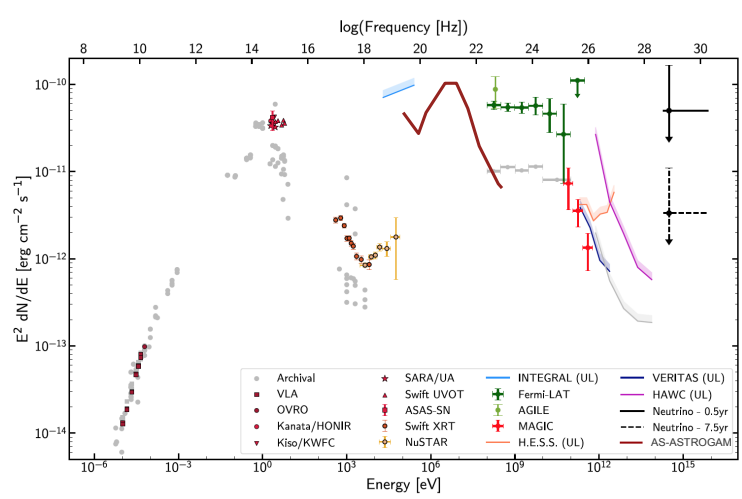

Figure 5: Spectral energy distribution of the blazar TXS 0506+056. Dark points are data taken during the flare; grey points correspond to non-flaring states. The All-Sky-ASTROGAM sensitivity (solid brown) is calculated for an effective exposure of 7 days, corresponding to the observations by Fermi-LAT and MAGIC during the flare.

ization, will be important to test a potential association between high-energy neutrino candidates and various classes of transient astrophysical events, and will continue the programs currently performed with the Swift and Fermi satellites.

Furthermore, it has been recently claimed that the gamma-ray transparency of astrophysical sources of neutrinos and gamma-rays coming from photoproduction of pions might be severely reduced [16]. This result consequently suggests a population of cosmic-ray accelerators invisible in $\mathrm{GeV}-$ $\mathrm{TeV}$ gamma rays but bright in the $\mathrm{MeV}$ domain (see e.g. [17]). All-Sky-ASTROGAM will provide a good sampling of their MeV light curves that will be used to search for neutrino counterparts. All-Sky-ASTROGAM should also observe $\sim 600$ GRBs during the first three years of its mission. Its sub-millisecond trigger and alert capabilities will enable to look for neutrino counterparts of GRBs in nearly real-time and will then take over from Swift, INTEGRAL and Fermi instruments. Finally, one of the yet unanswered questions is the nature of the process generating the observed cosmic neutrinos ( $p \gamma$ or $p p$ processes). If IceCube neutrinos are mainly produced by $p p$ interactions, their sources should significantly contribute to the Isotropic diffuse Gamma-Ray Background (IGRB) and their flux should be consistent with the total flux. Recent studies (see e.g. [18]) show that $p p$ models are in tension with the IGRB, disfavoring the $p p$ origin of the cosmic neutrino flux observed by IceCube. Further understanding the contribution of different source populations to the IGRB is therefore crucial. Measurement of spectral features in the $10-200 \mathrm{MeV}$ range with AllSky-ASTROGAM will help to constrain the population models of the IGRB and will consequently have an important impact on the interpretation of the multimessenger connection between gamma rays and neutrinos.

\section{Conclusions and perspectives}

All-Sky-ASTROGAM is designed to cover an energy range still poorly explored, betwenn $0.1 \mathrm{MeV}$ to a few hundreds $\mathrm{MeV}$. It has been conceived to operate in a maturing multi-messenger epoch, opening-up new exciting synergies. The mission data, inserted in a multiwavelength and multimessenger context, will allow addressing major topics of modern astrophysics, providing in- 
formation of significant interest to a broad astronomical community, in a decade of powerful observatories such as LIGO-VIRGO-KAGRA-INDIGO, SKA, ALMA, E-ELT, LSST, JWST, Athena, CTA, IceCube.

\section{References}

[1] Abbott, B.P., et al., 2017, ApJ Lett., 848, L12

[2] Aartsen, M.G., et al. (IceCube, Fermi-LAT, MAGIC Collaborations et al.), 2018, Science, 361, 6398

[3] De Angelis, A., Tatischeff, V., Tavani, M., Oberlack, U., et al., on behalf of the e-ASTROGAM Collaboration, 2017, Experimental Astronomy, 44, 25

[4] De Angelis, A., Tatischeff, V., Grenier, I., McEnery, J., Mallamaci, M., et al., 2018, J. High Energy Astrophysics, 19, 1

[5] Ackermann, M., et al. 2015, ApJ, 799, 1

[6] Fong, W., et al., 2014, ApJ, 780, 118

[7] Abbott, B. P., Abbott, R., Abbott, T. D., et al., 2017, Phys. Rev. Lett., 119, 161101

[8] Ghisellini, G., Lazzati, D., 1999, MNRAS, 309, 7

[9] Granot, J., 2002, ApJ, 570, 61

[10] Patricelli, B., et al., 2016, JCAP, 11, 56

[11] Savchenko, V., Ferrigno, C., Kuulkers, E., et al., 2017, ApJ, 848, L5

[12] Hotokezaka, K., et al., 2016, MNRAS, 459, 35

[13] Aartsen, M.G., et al. (IceCube collaboration), 2013, Science, 779, 132

[14] Aartsen, M. G., et al. (IceCube collaboration), 2018, Science, 361, 147

[15] Ansoldi, S., et al. (MAGIC collaboration), 2018, ApJ Lett., 863, L10

[16] Murase, K., et al., 2016, Phys. Rev. Lett., 116, 71101

[17] Senno, N., et al., 2016, Phys. Rev. D, 93, 83003

[18] Bechtol, K., et al., 2017, ApJ, 836, 47 